\title{
Visualization of the Autonomous Systems Interconnections with HERMES ${ }^{\star}$
}

\author{
Andrea Carmignani, Giuseppe Di Battista, Walter Didimo, \\ Francesco Matera, and Maurizio Pizzonia \\ Dipartimento di Informatica e Automazione, \\ Università di Roma Tre, via della Vasca Navale 79, \\ 00146 Roma, Italy. \\ \{carmigna, gdb, didimo, matera, pizzonia\}@dia.uniroma3.it
}

\begin{abstract}
Hermes is a system for exploring and visualizing Autonomous Systems and their interconnections. It relies on a three-tiers architecture, on a large repository of routing information coming from heterogeneous sources, and on sophisticated graph drawing engine. Such an engine exploits static and dynamic graph drawing techniques.
\end{abstract}

\section{Introduction and Overview}

Computer networks are an endless source of problems and motivations for the Graph Drawing and for the Information Visualization communities. Several systems aim at giving a graphical representation of computer networks at different abstraction levels and for different types of users. To give only some examples (an interesting survey can be found in [T]):

1. Application level: Visualization of Web sites structures, Web maps [1816], and Web caches [10].

2. Network level: Visualization of multicast backbones [22], internet traffic [23], routes, and interconnection of routers.

3. Data Link level: interconnection of switches and repeaters in a local area network [1].

We deal with the problem of exploring and visualizing the interconnections between Autonomous Systems. An Autonomous System (in the following AS) is a group of networks under a single administrative authority. Roughly speaking, an AS can be seen as a portion of Internet, and Internet can be seen as the totality of the ASes and their interconnections. Each AS is identified by an integer number.

A route is a path on the network that can be used to reach a specific set of (usually contiguous) IP addresses. A route is described by its IP addresses, its cost, and by the set of ASes that are traversed. Routes are "announced" from an

\footnotetext{
* Research supported in part by the Murst Project: "Algorithms for Large Data Sets". Hermes is presented at http://www.dia.uniroma3.it/ hermes.
}

J. Marks (Ed.): GD 2000, LNCS 1984, pp. 150-163 2001.

(C) Springer-Verlag Berlin Heidelberg 2001 
AS with messages like "through me you can reach a certain set of IP addresses, with a certain cost and traversing a certain set of other ASes".

In order to exchange route's information, the ASes adopt a network protocol called BGP (Border Gateway Protocol) 31. Such a protocol is based on a distributed architecture where border routers that belong to distinct adjacent ASes exchange information about the routes they know. The exchange of routing information between ASes is subject to routing policies; for example an AS can say "I do not want to be traversed by packets going to a certain other AS".

Several tools have been developed for analyzing and visualizing the Internet topology at the ASes level [912 2330. However, in our opinion, such tools are still not completely satisfactory both in the interaction with the user and in the effectiveness of the drawings. Some of them have the goal of showing large portions of Internet, but the maps they produce can be difficult to read (see e.g. 9]). Other tools point the attention on a specific AS, only showing that AS and its direct connections (see e.g. 23]).

In this work we describe a new system, called HeRMES, which allows to get several types of information on a specific AS and to explore and visualize the ASes interconnections. The main features of HeRMES are the following.

HeRmes has a three tiers architecture. The user interacts with a top-tier client which collects the user requests and forwards them to a middle-tier server. The server translates the requests into queries to a repository (the bottom tier). With the top-tier the user can explore and visualize the ASes interconnections, and several information about ASes and the BGP routing policies. The user interacts with a subgraph (called map) of the graph of all ASes interconnections. Each exploration step enriches the map with new ASes and connections (vertices and edges).

HERMES handles a large repository (about $50 \mathrm{MB}$ ). The repository is updated off-line from a plurality of sources [24]: APNIC, ARIN, BELL, CABLE\&WIRE, CANET, MCI, RADB, RIPE, VERIO. The data in the repository are used from HERMES to construct the ASes interconnection graph.

The middle-tier server of HERMES encapsulates a graph drawing module that computes the drawing of the ASes interconnections already explored at a specific time. Such a module is based on the GDToolkit library [21] and has the following main features:

- Its basic drawing convention is the podevsnef [20] model for orthogonal drawings having vertices of degree greater than four. However, since the handled graphs have often many vertices (ASes) of degree one connected with the same vertex, the podevsnef model is enriched with new features for representing such vertices.

- It is equipped with two different graph drawing algorithms. In fact, at each exploration step the map is enriched and hence it has to be redrawn. Depending on the situation, the system (or the user) might want to use a static or a dynamic algorithm. Of course, the dynamic and the static algorithms have advantages and drawbacks. The dynamic algorithm allows to preserve the mental map of the user [1927] but can lead, after a certain number of 
exploration steps, to drawings that are less readable than those constructed with a static algorithm.

- The static algorithm is based on the topology-shape-metrics approach [14] and exploits recent compaction techniques that can draw vertices with any prescribed size [13]. The topology-shape-metrics approach has been shown to be very effective and reasonably efficient in practical applications [15].

- The dynamic algorithm is a new dynamic graph drawing algorithm that has been devised according to three main constraints. (1) It had to be completely integrated within the topology-shape-metrics approach in such a way to be possible to alternate its usage with the usage of the static algorithm. (2) It had to be consistent with the variation of the podevsnef model used by HeRMES. (3) It had to allow vertices of arbitrary size. Several algorithms have been recently proposed in the literature on dynamic graph drawing algorithms. In [4] a linear time algorithm for orthogonal drawings is presented, where the position of the vertices cannot be changed after the initial placement. In [29] four different scenarios for interactive orthogonal graph drawings are studied. Each scenario defines the changes allowed in the common part of two consecutive drawings. In [7] it is described an interactive version of Giотто [33]; it allows to incrementally add vertices and edges to an orthogonal drawing so that the shape of the common part of two consecutive drawings is preserved and the number of bends is minimized under this constraint. In [5] it is presented a dynamic algorithm for orthogonal drawings that allows to specify the relative importance of the number of bends vs. the number of changes between two consecutive drawings. Other algorithms for constructing drawings of graphs incrementally, while preserving the mental map of the user, are for example [2611/28]. Also, in [6] it is presented a study on different metrics that can be used to evaluate the changes between drawings in an interactive scenario. However, as far as we know, none of the cited dynamic algorithms enforces all the constraints (1)-(3).

The paper is organized as follows. In Section 2 we explain how the user interacts with HeRMES and provide a high level description of the functionalities of the system. In Section 3 we give some details about the three tiered architecture of Hermes. Section 4 shows the results of a study on the ASes interconnection graph we have performed before choosing the graph drawing algorithms to apply in Hermes. In particular we give measures on the local density and on the average degree of the vertices. In Section 5$]$ we describe the drawing convention and the algorithms.

\section{Using Hermes}

The user interacts with HeRmes through an ASes subgraph. An ASes subgraph (also called map) is a subgraph of the graph of all ASes and their interconnections. A map is initially constructed using two possible starting primitives. AS selection: An AS is chosen. The obtained map consists of such an AS plus all 
the ASes that are connected to it. See Fig. 1(a) Routes selection: A set of routes is selected. The user has two possibilities: (1) selection of all the routes traversing a specific AS (see Fig. 2); (2) selection of all the routes traversing a specific pair of ASes. The obtained map consists of all the ASes and connections traversed by the selected routes. The user has also the option of cutting the map pruning all the ASes that are after (before) the selected AS in the routes propagation (see Figs. 2(a) and 2(b)).

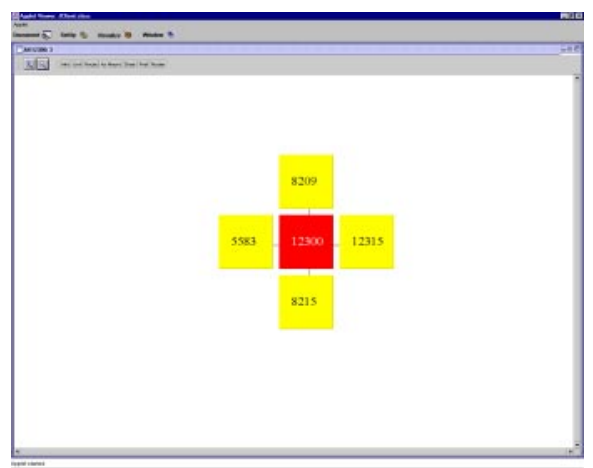

(a) Selection of AS 12300.

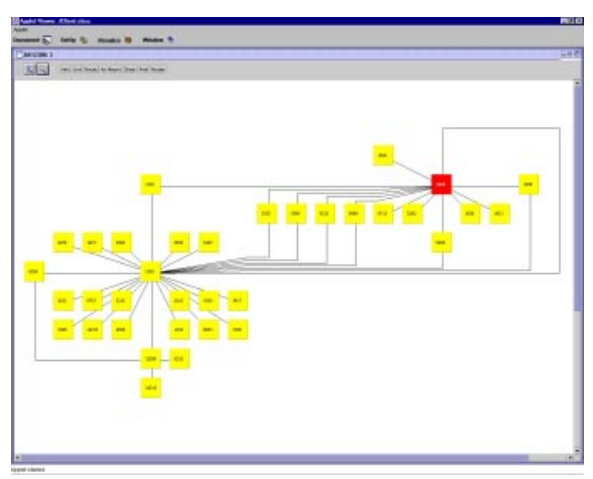

(c) Exploration of AS 5484.

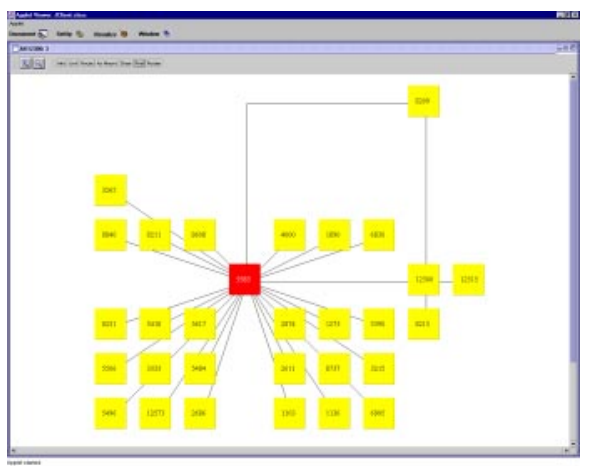

(b) Exploration of AS 5583.

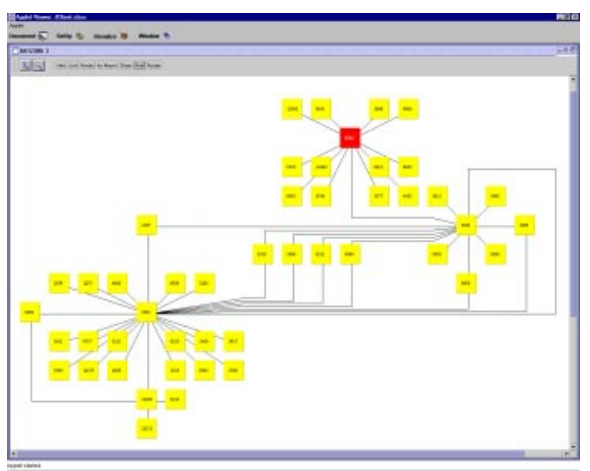

(d) Exploration of AS 6715.

Fig. 1. Exploration steps in the ASes graph. The selected AS is always drawn red.

The user can explore and enrich the map by using the following primitive. AS exploration: an AS $u$ among those displayed in the current map is selected. The current map is augmented with all the ASes that are connected to $u$. Further, for each AS $v$ connected to $u$ an edge $(u, v)$ is added. Observe that, according 


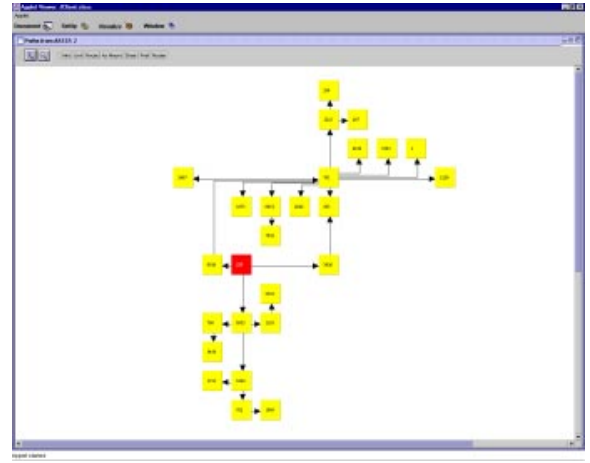

(a) Map containing only the ASes after AS 137.

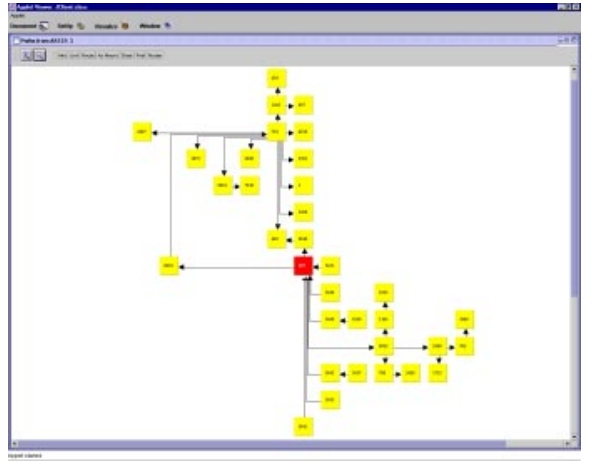

(b) Map containing all the traversed ASes.

Fig. 2. Selection of the routes traversing AS 137.

to this definition, a map is a subgraph of the ASes interconnection graph but it is not an induced subgraph.

Fig. 1 shows a sequence of exploration primitives applied to the map of Fig. 1(a) ASes 5583, 5484, and 6715 are explored in Fig's. 1(b), 1(c), and 1(d) respectively. Fig. 1highlights several features of HeRMES. HeRMES can construct new drawings either using a static or a dynamic graph drawing algorithm. Observe how the drawing of Fig. 1(b) has been constructed with a dynamic algorithm starting from the drawing of Fig. 1(a), The drawing of Fig. 1(d) has been constructed with a dynamic algorithm starting from the drawing of Fig. 1(c) Conversely, the drawing of Fig. 1(c) is obtained with a static algorithm. The choice of the algorithm to apply can be done by the system (see Section 5) or forced by the user. Since the ASes degree can be large (see Section 4), in the project of the drawing algorithms of HERMES, special attention has been devoted to the representation of vertices of high degree. Fig. 1 shows how the vertices of degree one are placed around their adjacent vertices. The user can also select a more traditional way for displaying such vertices, according to the simplepodevsnef model described in [13] (see Fig. 2). A more complex map obtained with Hermes is depicted in Fig. 3 It contains more than 150 ASes.

Working on a map, independently on the way it has been obtained, the user can get several information on any AS:

General Info : Name, Maintainers, Connections, and Description. See Fig. 4. Routing Policies : For each connected AS, an expression describing the policy and its cost. See Fig. 4 This is possible both for in and for out policies. The default AS is also displayed.

Internal Routers : List of the known border routers with the IP-numbers of the interfaces. Peering sessions with other routers are displayed. 


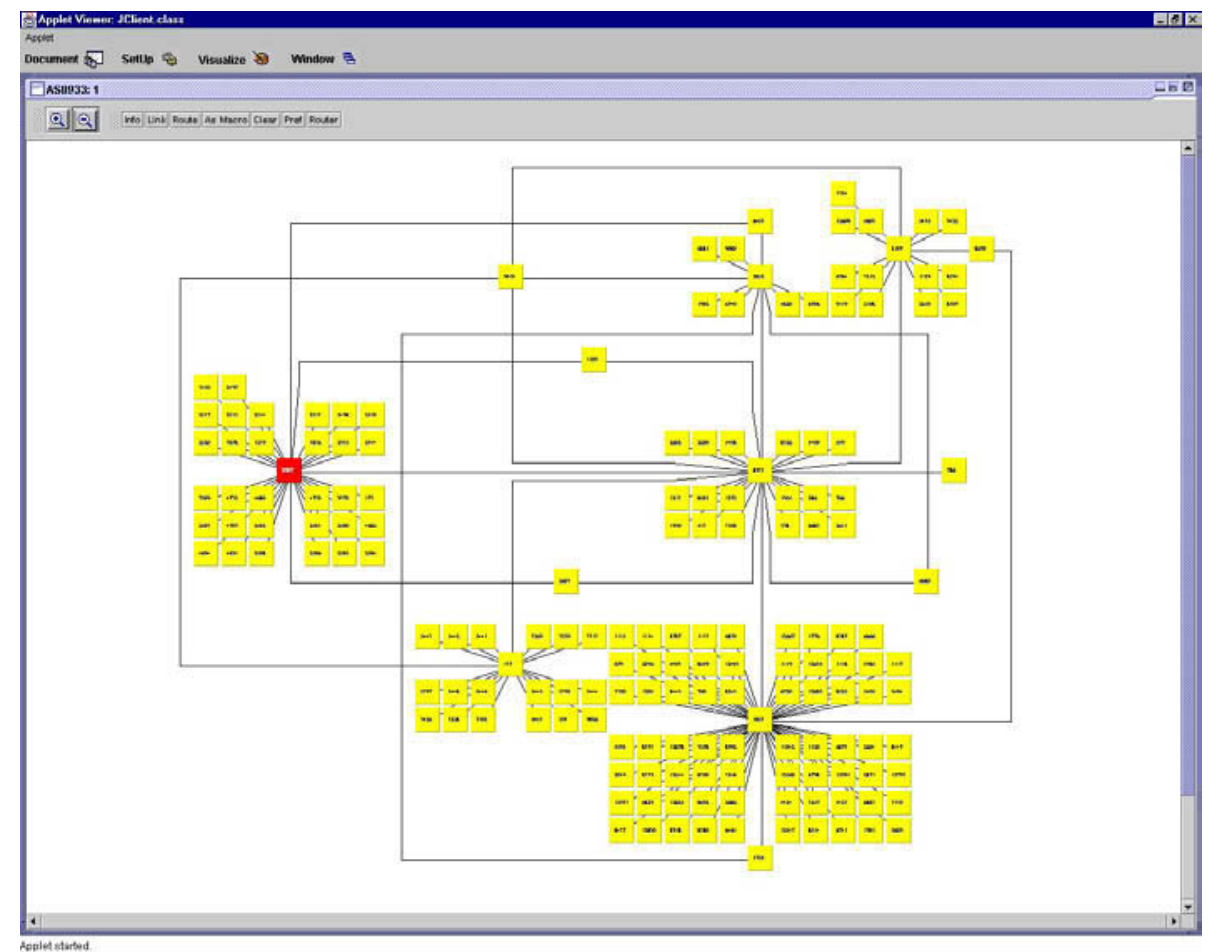

Fig. 3. A map obtained with several exploration steps.

Routes : List of the routes originated by the AS. It is also possible to visualize the propagation of a given route in the ASes composing the map.

AS Macros : List of the macros 2] including the AS.

\section{A Three Tiers Architecture}

The architecture of HERMES is three tiered. The user interacts with a top-tier client which is in charge of collecting user requests and showing results. The requests are forwarded by the client to a middle-tier server which is in charge to process the raw data extracted from a repository (bottom tier).

The client is a multi-document GUI-based application. It allows the user to carry-on multiple explorations of the ASes graph at the same time. The Java technology has been used to ensure good portability. Snapshots of the GUI have been shown in Section 2 ,

The repository is updated off-line from a plurality of sources. At the moment we access the following databases adopting, for representing data, the RIPE-181 language [28]: ANS, APNIC, ARIN, BELL, CABLE\&WIRE, CANET, MCI, 


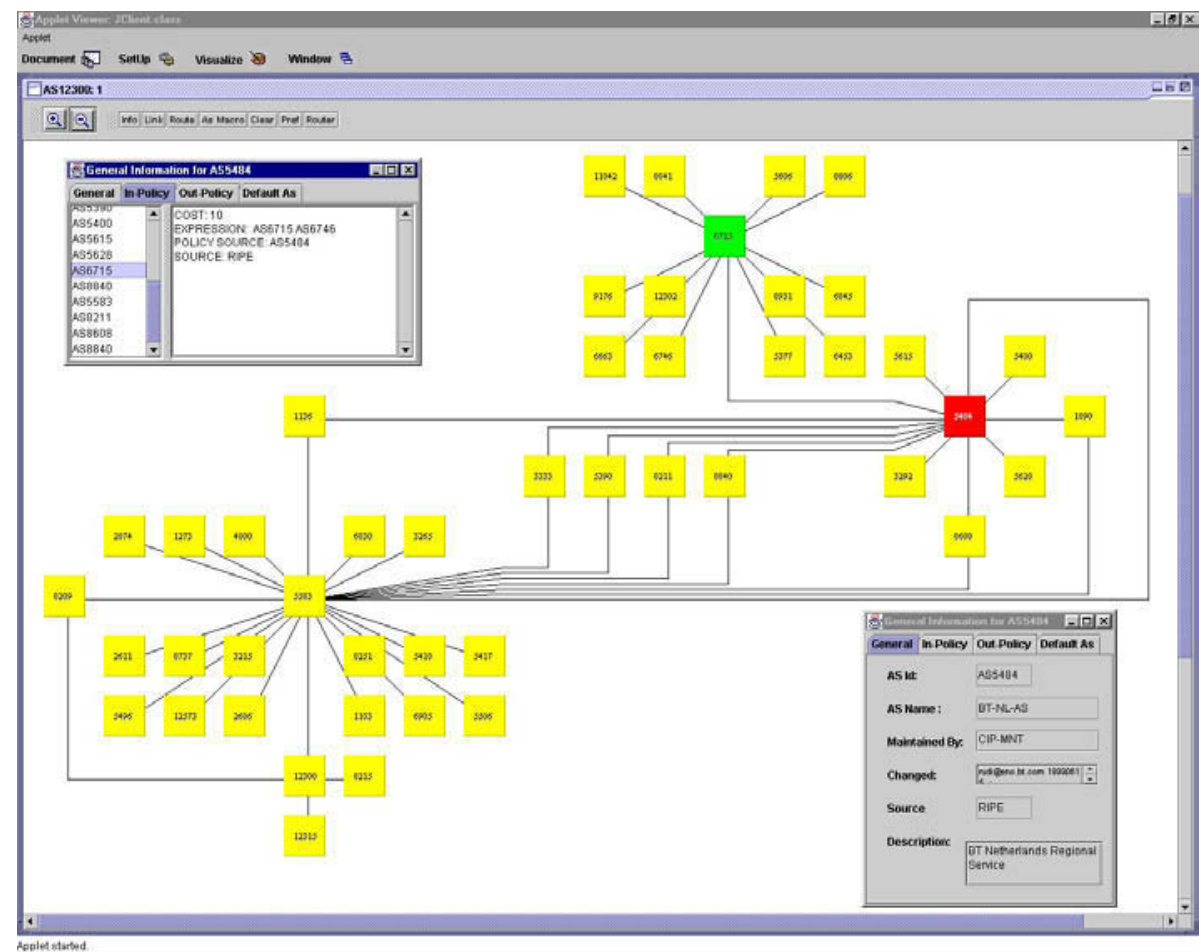

Fig. 4. General Info and routing policies for AS 5484.

RADB, RIPE, VERIO. Further, we access the routing BGP data provided by the Route views project of the Oregon University [25]. However, the repository is easily extensible to other data sources.

Data are filtered so that only the information used by HERMES are stored in the database, but no consistency check or ambiguity removal is performed in this stage. The overall size of the repository is about $50 \mathrm{MB}$. The adopted DBMS technology is currently mysql.

The crucial part of the system is mainly located in the middle-tier. The top tier requests two types of service to the middle tier. General info services: the top tier queries about ASes, routes, and path properties. Topology services: the top tier queries for a new exploration and gets back a new map.

Info services requests are independent each other and hence are independently handled by the middle-tier. On the contrary, topology services requests are always part of a drawing session. Each client may open one or more drawing sessions. Each drawing session is associated with a map that can be enriched by means of exploration requests. 
Info services requests are directly dispatched to a mediator. The mediator module is in charge to retrieve the data from the repository and to remove ambiguities on-the-fly.

Topology services requests are handled by the kernel of the system. It gets information from the mediator and inserts new edges and vertices into the map. The drawing is computed by the drawing engine module (see Section 5). The drawing engine encapsulates GDToolkit [21].

\section{AS Interconnection Data from a Graph Drawing Perspective}

In order to devise effective graph drawing facilities for HERMES, we have analyzed the ASes and their interconnections considering them as a unique large graph $G$. The data at our disposal show the following structure for $G$.

The number of vertices of $G$ is 6,849 , while the number of edges is 27,686 . Fig. 5(a) illustrates the distribution of the degree of the vertices. The figure shows that while there are many vertices (about $75 \%$ ) with degree less or equal than 4 , there are also several vertices whose degree is more than 100 . For improving the readability of the chart, we have omitted two vertices with degree 862 and 1, 044, respectively. Further, consider that $G$ contains 473 isolated vertices.

The density of $G$ is 4.04 . However, the "local" density can be much greater. In order to estimate such a local density, we have computed, for each vertex $v$, the density of the subgraph induced by the vertices adjacent to $v$. We call such graphs local graphs. Fig. 5(b) illustrates the distribution of the densities of the local graphs. From the figure it is possible to observe that about $5 \%$ of the local graphs have density greater than 10 .

We have also tried to estimate the probability, for a user that explores $G$, to encounter a portion of $G$ that is locally dense. Fig. 5(c) shows, for each value $d$ of density, what is the percentage of vertices that are adjacent to a vertex whose local graph has density at least $d$. Note that more than $30 \%$ of the vertices are adjacent to a vertex whose local graph has density at least 10 .

Concerning connectivity, the graph has 480 connected components, including the above mentioned 473 isolated vertices. One of them has 6,360 vertices; each of the remaining 6 components has less than 6 vertices.

\section{Drawing Conventions and Algorithmic Issues}

At each exploration step, HeRmes computes a new drawing. Namely, when the user selects in the map a new vertex $v$, all the vertices and edges connected to $v$ are added to the map, and such a map is redrawn. We use, depending on the specific situation, two different drawing algorithms.

Static algorithm The current map is completely redrawn, after the new vertices and edges have been added. 


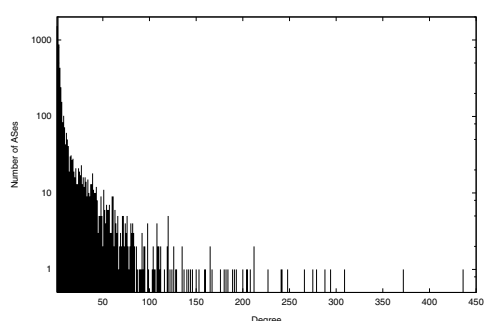

(a) AS degree distribution

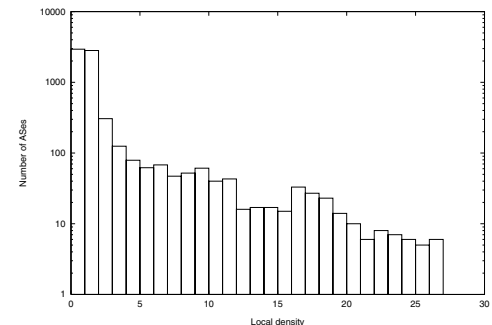

(b) AS density distribution

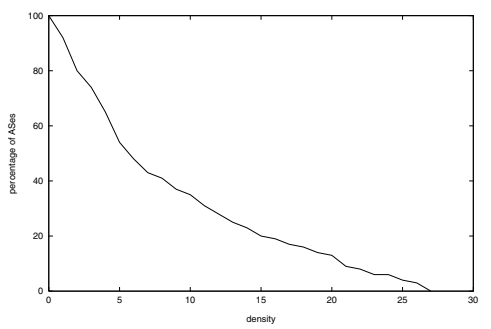

(c) Percentage of ASes adjacent to an AS whose local graph has at least a given value of density

Fig. 5. Several aspects of the density of the ASes graph.

Dynamic algorithm The new vertices and edges are added to the current drawing in such a way that the shape of the existing edges and the position of the existing vertices and bends are preserved "as much as possible".

Of course, the dynamic and the static algorithms have advantages and drawbacks. The dynamic algorithm allows to preserve the mental map of the user but can lead, after a certain number of exploration steps, to drawings that are less readable than those constructed with the static algorithm. In fact, the dynamic algorithm makes use of local optimization strategies. The optimization strategies of the static algorithm are global and more effective. However, with the static algorithm, the new drawing can be quite different from the previous one and the user's mental map can be lost.

Because of the above motivations, we automatically choose between the static algorithm and the dynamic algorithm, depending on the number and the kind of egdes and vertices that are added when a new vertex is explored. However, the user can always force the system to apply one of the two algorithms. Namely, suppose $v$ is the vertex the user wants to explore. The choice of the drawing 
algorithm to apply is done by calculating an exploration cost for $v$ and comparing such a cost with a treshold that can be set-up in a configuration menu. The exploration cost is computed as follows. For any new edge the cost is 1 . For any vertex that had degree 1 in the old graph and whose degree is increased in the new graph the cost is 0.5 . An extra cost is also added as a function of the number of times the dynamic algorithm has been invoked before. The exploration cost is a very rough estimate of the efficiency and effectiveness of the dynamic algorithm with respect to the new exploration. The reason why vertices with degree 1 are treated in a special way will be clear in the description of the algorithms.

The static algorithm we use consists of the following steps.

Degree-one Vertex Removal Vertices of degree one are temporarily removed.

Planarization A standard planarization [14 technique is applied.

Orthogonalization and Compaction We apply a variation of the technique presented in [13] for constructing orthogonal drawings (in the simplepodavsnef model) with vertices of prescribed size. The box representing a vertex $v$ is a rectangle. Edges incident on $v$ can incide the box only in the middle points of the sides. The length of the sides are chosen in such a way to have enough space to accommodate all the vertices that have been temporarily removed in the first step and that were adjacent to $v$.

Degree-one Vertex Re-insertion Each box representing a vertex $v$ is partitioned into nine rectangles arranged into three rows and three columns. Denote them as $B_{i, j}$. Rectangle $B_{2,2}$ is used for drawing $v$. Rectangles $B_{1,1}$, $B_{1,3}, B_{3,1}$, and $B_{3,3}$ are used for drawing the degree-one vertices adjacent to $v$. Their incident edges are represented with straight-line segments, possibly overlapping other degree-one vertices. Actually, they are drawn on the back of the vertices. Rectangles $B_{1,2}, B_{2,1}, B_{3,2}$, and $B_{2,3}$ are used for hosting the connections of $v$ to the other vertices. See Fig. 6 .

The dynamic drawing algorithm allows to apply three primitives on the current map. New-Edge( $(\mathbf{u}, \mathbf{v})$ : a new edge is added to the map between the two vertices $u$ and $v$; vertices $u$ and $v$ must be already in the current map. Attach$\operatorname{Vertex}(\mathbf{u})$ : a new vertex $v$ is added to the map and connected to $u$ with a new

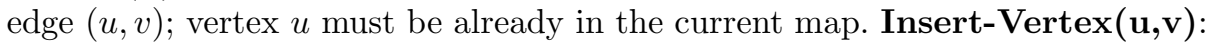
a new vertex is added to the map by splitting edge $(u, v)$; edge $(u, v)$ must be already in the current map.

The algorithm computes the position of the new vertices and edges trying to optimize several aesthetic measures (number of crossings, number of bends, and edge length) at the same time, depending on the costs the user has chosen for each of them. The obtained drawing is guaranteed to have the same shape of the starting one, for the common parts.

In the following we give some details about the used data structure and how the three drawing primitives work. Vertices of degree one are temporarily absorbed into their incident vertices, with the techniques described above.

We maintain a copy of the map with the following main extra features: 


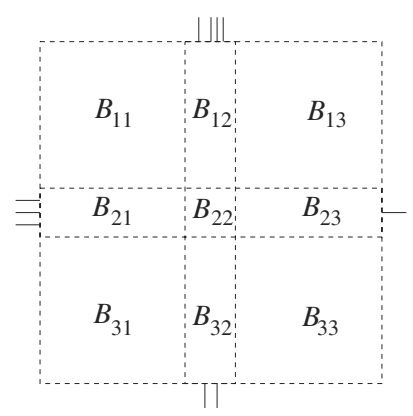

(a)

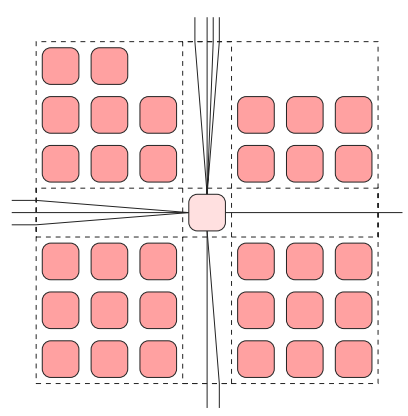

(b)

Fig. 6. Using a box to represent a vertex. (a) The nine regions. (b) Using the nine regions to reinsert degree-one vertices.

- All the crossings and bends of the map are replaced by dummy vertices, so that the topology of the drawing becomes planar.

- The map is simplified so that all vertices have degree less or equal than four. This is done with a standard technique adopted in the podevsnef model [3], where all the edges incident on the same vertex from the same side are collapsed into one single edge. See Fig. 7(a). A thickness is associated with each of the new edges, representing the number of edges it replaces. The transformation is recursively applied to all the vertices.

- New edges are added to the map for decomposing each face (including the external one) into rectangles, with the linear time algorithm described in [32]. We call dashed the new edges and solid the edges of the original map.

- An incidence network $D$ is constructed. Such a network is defined as follows (see Fig. 7(b)): (1) The nodes of $D$ are the (solid and dashed) edges of the map. (2) Nodes corresponding to solid edges have associated a cost equal to their thickness multiplied by a constant $\chi$ (see Fig. $7(\mathrm{a})$ ). Nodes corresponding to dashed edges have cost equal to zero. Intuitively, the cost associated with an edge represents the cost of a crossing involving that edge. (3) An arc is added to $D$ for each pair of edges of the map sharing a face. Fig. 7(b) shows three arcs of $D$. (4) An arc between two horizontal (vertical) edges that lie on different sides of the same face has a cost that is equal to their vertical (horizontal) distance multiplied by a constant $\lambda$. See Fig. $7(\mathrm{~b})$ Intuitively, the cost associated with an arc of this type represents a lower bound on the length of a possible new edge that follows the arc. (5) An arc between a horizontal and a vertical edge has a cost that is equal to the orthogonal distance between the centers of the edges multiplied by a constant $\lambda$ plus $\beta$. See Fig. 7(b). Intuitively, the cost associated with an arc of this type represents a lower bound on the length of a possible new edge that follows the arc plus a cost for a bend. (6) An arc between two horizontal 
(vertical) edges that lie on the same side of the same face has a cost that is equal to the distance between the centers of the edges plus 2 (multiplied by a constant $\lambda$ ) plus $2 \beta$. See Fig. $7(\mathrm{~b})$. Intuitively, the cost associated with an arc of this type represents a lower bound on the length of a possible new edge that follows the arc plus a cost for two bends. (7) Intuitively, $\chi, \beta$, and $\lambda$, represent the costs for one cross, one bend, and one unit of length, respectively. Their values can be set-up by the user. The ratios between $\chi$, $\beta$, and $\lambda$ may determine different behaviors of the algorithm.

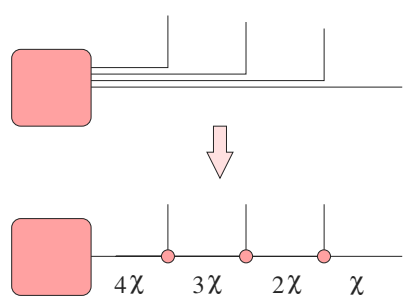

(a) Collapsing the edges incident on the same side of a vertex.

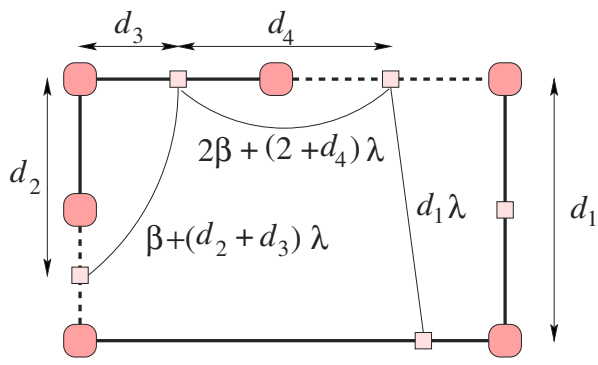

(b) Nodes (little squares) and arcs of the incidence network.

Fig. 7. Illustration of the dynamic algorithm.

Primitives New-Edge, Attach-Vertex, and Insert-Vertex are implemented as follows.

New-Edge $(\mathbf{u}, \mathbf{v}):$ In the case $u$ and $v$ are not on the same face two temporary nodes are added to $D$ representing $u$ and $v$. Also, temporary arcs are added to $D$ between $u$ and the nodes representing its incident edges. The same is done for $v$. The temporary arcs have zero cost.

A shortest path between $u$ and $v$ is computed. Such a path determines the route and the shape of the new edge. Namely, the new edge is inserted in the map following the arcs of the shortest path. The temporary nodes and arcs are removed an the new faces originated by the new edge are decomposed into rectangles.

In the case $u$ and $v$ are on the same face a simpler technique (not discussed here for brevity) is adopted.

$\operatorname{Attach}-\operatorname{Vertex}(\mathbf{u})$ : A local evaluation of the edges incident on $u$ is performed. The new edge is put preferably either on a direction around $u$ where there is no incident edge or on a dashed edge.

Insert-Vertex $(\mathbf{u}, \mathbf{v}):$ Edge $(u, v)$ is just split into two pieces. If the edge has a bend we put the new vertex preferably on that bend. 
Once a primitive has been performed, the expansion technique described above is applied to make room for the vertices of degree one that were temporarily absorbed into their incident vertices.

Observe that $D$ can have a number of arcs that is quadratic in the number of edges of the map. However, it is possible to see that $D$ can be simplified to an equivalent net with a linear number of arcs.

Acknowledgements. We are grateful to Sandra Follaro and Antonio Leonforte for their fundamental contribution in the implementation of the dynamic algorithm. We are also grateful to Andrea Cecchetti for useful discussion on the repository.

\section{References}

1. Aprisma. Spectrum. On line. http://www.aprisma.com.

2. T. Bates, E. Gerich, L. Joncheray, J. M. Jouanigot, D. Karrenberg, M. Terpstra, and J. Yu. Representation of ip routing policies in a routing registry. On line, 1994. ripe-181, http://www.ripe.net, rfc 1786.

3. P. Bertolazzi, G. Di Battista, and W. Didimo. Computing orthogonal drawings with the minimum numbr of bends. IEEE Transactions on Computers, 49(8), 2000.

4. T. C. Biedl and M. Kaufmann. Area-efficient static and incremental graph darwings. In R. Burkard and G. Woeginger, editors, Algorithms (Proc. ESA '97), volume 1284 of Lecture Notes Comput. Sci., pages 37-52. Springer-Verlag, 1997.

5. U. Brandes and D. Wagner. Dynamic grid embedding with few bends and changes. In K.-Y. Chwa and O. H. Ibarra, editors, ISAAC'98, volume 1533 of Lecture Notes Comput. Sci., pages 89-98. Springer-Verlag, 1998.

6. S. Bridgeman and R. Tamassia. Difference metrics for interactive orthogonal graph drawing algorithms. In S. H. Withesides, editor, Graph Drawing (Proc. GD '98), volume 1547 of Lecture Notes Comput. Sci., pages 57-71. Springer-Verlag, 1998.

7. S. S. Bridgeman, J. Fanto, A. Garg, R. Tamassia, and L. Vismara. InteractiveGiotto: An algorithm for interactive orthogonal graph drawing. In G. Di Battista, editor, Graph Drawing (Proc. GD '97), volume 1353 of Lecture Notes Comput. Sci., pages 303-308. Springer-Verlag, 1998.

8. M. Bukowy and J. Snabb. RIPE NCC database documentation update to support RIPE DB ver. 2.2.1. On line, 1999. ripe-189, http://www.ripe.net.

9. CAIDA. Otter: Tool for topology display. On line. http://www.caida.org.

10. CAIDA. Plankton: Visualizing nlanr's web cache hierarchy. On line. http://www.caida.org.

11. R. F. Cohen, G. Di Battista, R. Tamassia, and I. G. Tollis. Dynamic graph drawings: Trees, series-parallel digraphs, and planar ST-digraphs. SIAM J. Comput., 24(5):970-1001, 1995.

12. Cornell University. Argus. On line. http://www.cs.cornell.edu/cnrg/topology_aware/discovery/argus.html.

13. G. Di Battista, W. Didimo, M. Patrignani, and M. Pizzonia. Orthogonal and quasi-upward drawings with vertices of prescribed sizes. In J. Kratochvil, editor, Graph Drawing (Proc. GD '99), volume 1731 of Lecture Notes Comput. Sci., pages 297-310. Springer-Verlag, 1999.

14. G. Di Battista, P. Eades, R. Tamassia, and I. G. Tollis. Graph Drawing. Prentice Hall, Upper Saddle River, NJ, 1999. 
15. G. Di Battista, A. Garg, G. Liotta, R. Tamassia, E. Tassinari, and F. Vargiu. An experimental comparison of four graph drawing algorithms. Comput. Geom. Theory Appl., 7:303-325, 1997.

16. G. Di Battista, R. Lillo, and F. Vernacotola. Ptolomaeus: The web cartographer. In S. H. Withesides, editor, Graph Drawing (Proc. GD '98), volume 1547 of Lecture Notes Comput. Sci., pages 444-445. Springer-Verlag, 1998.

17. M. Dodge. An atlas of cyberspaces. On line. http://www.cybergeography.com/atlas/atlas.html.

18. P. Eades, R. F. Cohen, and M. L. Huang. Online animated graph drawing for web navigation. In G. Di Battista, editor, Graph Drawing (Proc. GD '97), volume 1353 of Lecture Notes Comput. Sci., pages 330-335. Springer-Verlag, 1997.

19. P. Eades, W. Lai, K. Misue, and K. Sugiyama. Preserving the mental map of a diagram. In Proceedings of Compugraphics 91, pages 24-33, 1991.

20. U. Fößmeier and M. Kaufmann. Drawing high degree graphs with low bend numbers. In F. J. Brandenburg, editor, Graph Drawing (Proc. GD '95), volume 1027 of Lecture Notes Comput. Sci., pages 254-266. Springer-Verlag, 1996.

21. GDToolkit:. Graph drawing toolkit. On line. http://www.gdtoolkit.com.

22. B. Huffaker. Tools to visualize the internet multicast backbone. On line. http://www.caida.org.

23. IPMA. Internet performance measurement and analysis project. On line. http://www.merit.edu/ipma.

24. Merit Network, Inc. Radb database services. On line. http://www.radb.net.

25. D. Meyer. University of oregon route views project. On line. http://www.antc.uoregon.edu/route-views.

26. K. Miriyala, S. W. Hornick, and R. Tamassia. An incremental approach to aesthetic graph layout. In Proc. Internat. Workshop on Computer-Aided Software Engineering, 1993.

27. K. Misue, P. Eades, W. Lai, and K. Sugiyama. Layout adjustment and the mental map. J. Visual Lang. Comput., 6(2):183-210, 1995.

28. S. North. Incremental layout in DynaDAG. In F. J. Brandenburg, editor, Graph Drawing (Proc. GD '95), volume 1027 of Lecture Notes Comput. Sci., pages 409418. Springer-Verlag, 1996.

29. A. Papakostas and I. G. Tollis. Interactive orthogonal graph drawing. IEEE Transactions on Computers, 47(11):1297-1309, 1998.

30. C. Rachit. Octopus: Backbone topology discovery. On line. http://www.cs.cornell.edu/cnrg/topology_aware/topology/Default.html.

31. Y. Rekhter. A border gateway protocol 4 (bgp-4). IETF, rfc 1771.

32. R. Tamassia. On embedding a graph in the grid with the minimum number of bends. SIAM J. Comput., 16(3):421-444, 1987.

33. R. Tamassia, G. Di Battista, and C. Batini. Automatic graph drawing and readability of diagrams. IEEE Trans. Syst. Man Cybern., SMC-18(1):61-79, 1988. 